\title{
Minimax state estimation for linear discrete-time differential-algebraic equations
}

\author{
Sergiy M.Zhuk \\ Department of System Analysis and Deicision Making Theory, Taras Shevchenko National University of Kyiv, Ukraine
}

\begin{abstract}
This paper presents a state estimation approach for an uncertain linear equation with a non-invertible operator in Hilbert space. The approach addresses linear equations with uncertain deterministic input and noise in the measurements, which belong to a given convex closed bounded set. A new notion of a minimax observable subspace is introduced. By means of the presented approach, new equations describing the dynamics of a minimax recursive estimator for discrete-time non-causal differentialalgebraic equations (DAEs) are presented. For the case of regular DAEs it is proved that the estimator's equation coincides with the equation describing the seminal Kalman filter. The properties of the estimator are illustrated by a numerical example.
\end{abstract}

Key words: Robust estimation; Descriptor systems; Optimization under uncertainties; Set-membership estimation; Minimax 1991 MSC: 93E11 93E10 60G35

\section{Introduction}

The importance of models described by DAEs (or descriptor systems) in economics, demography, mechanics and engineering is well known [13]. Here, motivated by further applications to linear DAEs, we present a state estimation approach for linear deterministic models described by an abstract linear equation in a Hilbert space. Our approach is based on ideas underlying $H_{2} / H_{\infty}$ filtering $[4,3]$ and set-membership state estimation $[7,14,16,12,15]$.

$\mathrm{H}_{2}$-estimators like Kalman or Wiener filters $[4,1]$ give estimations of the system state with minimum error variance. The $\mathrm{H}_{2}$-estimation problem for linear timevariant DAEs was studied in [17] without restricting the DAE's matrices. The resulting algorithm requires the calculation of the so-called " 3 -block matrix pseudoinverse". In [10] the authors introduced explicit formulas for the 3-block matrix pseudoinverse and derived a recurrence filter, assuming a special structure for the DAE's matrices. A brief overview of steady-state $\mathrm{H}_{2}$ estimators is presented in [8].

$H_{\infty}$ estimators minimize a norm of the operator mapping unknown disturbances with finite energy to filtered errors [3]. We stress that the $H_{\infty}$ estimator coincides

\footnotetext{
* This paper was presented at IFAC Workshop CAO09, Jyvaskyla, Finland, May 6-9, 2009. Corresponding author S. Zhuk. Tel. +38050-52-59138. Fax +38044-57-56684.
}

Email address: beetle@unicyb.kiev.ua (Sergiy M.Zhuk). with a certain Krein space $H_{2}$ filter [19]. The $H_{\infty}$ filtering technique was applied to linear time-invariant DAEs with regular matrix pencils in [22].

A basic notion in the theory of set-membership state estimation is that of an a posteriori set or informational set. This notion has roots in control theory [4]. By definition, it is the set of all possible state vectors $\varphi$, that are consistent with a measured output $y$, provided that an uncertain input $f$ and measurement error $\eta$ belong to some bounded set $\mathscr{G}$. We will be interested in the case when the state $\varphi \in \mathcal{H}$ obeys an abstract linear equation $L \varphi=f$, provided $y=H \varphi+\eta,(f, \eta) \in \mathscr{G}$, where $\mathscr{G}$ is a bounded closed convex subset of an abstract Hilbert space. The problem is to find an estimation $\hat{\varphi}$ of $\varphi$ with minimal worst-case error. This problem was previously considered in $[16,14]$. Due to [16] a vector $\hat{\varphi}$ is called a linear minimax a-posteriori estimation (or a central algorithm due to [14]) iff $\forall \ell \in \mathcal{H}$

$$
\langle\ell, \hat{\varphi}\rangle=\left(\sup _{\mathscr{G}(y)}\langle\ell, \varphi\rangle+\inf _{\mathscr{G}(y)}\langle\ell, \varphi\rangle\right) / 2
$$

provided that an a posteriori set $\mathscr{G}(y):=\{\varphi:(L \varphi, y-$ $H \varphi) \in \mathscr{G}\}$ is a bounded convex subset of the Hilbert space $\mathcal{H}$. Note that if there exists $\varphi_{0}$ so that $L \varphi_{0}=0$, $H \varphi_{0}=0$, then $\sup _{\mathscr{G}(y)}\langle\ell, \varphi\rangle=+\infty$ for some $\ell$. Thus, the above approach does not work if $L$ is non-injective. In this paper we generalize the approach of $[14,16]$ to linear equations with non-injective $L$. Futher generalization is presented in [25]. 
The main contribution of this paper is a new notion of a minimax observable subspace $\mathcal{L}$ for the pair $(L, H)$ (Definition 1). It is useful when one needs to evaluate a priori how far the estimation $\hat{\varphi}$ is from a "real" state $\varphi$ in the worst case, provided $\hat{\varphi}$ is constructed from the measurements $y$. Due to Proposition 1, the worst-case estimation error is finite iff $\mathcal{L}=\mathcal{H}$; otherwise $\hat{\varphi}$ may be too far from a "real" state $\varphi$ for some directions $\ell \in \mathcal{H}$, even for bounded $f$ and $\eta$. In fact, given $y$, we can provide an estimation with finite worst-case error for the projection of $\varphi$ onto $\mathcal{L}$ only. Thus $\mathcal{L}$ is an analog of the observable subspace $[4$, p.240] for the pair $(L, H)$ in the context of set-membership state estimation.

The introduced notion allows the generalization of ideas from $[14,16,21]$ to non-injective linear mappings, in particular for the case $L \varphi(t)=\left((F \varphi)_{t}-C(t) \varphi(t), F \varphi\left(t_{0}\right)\right)$ with $F \in \mathbb{R}^{m \times n}$ which arise in the state estimation for linear continuous non-causal ${ }^{1}$ DAEs [24]. As a consequence, one can apply the minimax framework, originally developed $[7,5]$ for DAEs $(F=E$ in the linear case) with bounded uncertainties, to DAEs [25] with unbounded inputs (see example in Section 3).

In order to stress connections with $H_{\infty}$ approach, we note that the minimax framework $[7,5]$ incorporates the set-membership state estimation and $H_{\infty}$ filtering for ODEs by application of dynamic programming [2] to the informational state $X(\tau)$ : for linear ODE the worst-case estimation is set to be the Tchebysheff center of $X(\tau)$. Although we derive the estimation from the minimization of the worst-case error, as it is stated in Definition 1 , our approach (for the ellipsoidal bounding set $\mathscr{G}$ and causal $^{2}$ DAEs) results in the same estimation and error as in [7]. Thus, the $\ell$-minimax estimation gives a proper generalization of the recurrence algorithm from [7] to the case when $X(\tau)$ may be unbounded.

We illustrate the benefits of the new notion by introducing a minimax recursive estimator for discrete-time non-causal DAEs: it works for non-causal DAEs unlike $[10,8,22,23]$ and for the regular case it coincides with one proposed in [10] (Corollary 1). In addition, the minimax observability subspace allows one to identify the observable (in the minimax sense) part of the state with respect to given measurements. Computing the index of non-causality, one can a priori check how good connections between observations and state are: models with zero index are fully observable while models with non-zero index have an unobservable part in the state.

This paper is organized as follows. In Section 2 we give definitions (Definition 1) of the minimax estimation, error and observable subspace for abstract linear equations and we construct the estimation for a convex bounded $\mathscr{G}$, in particular for an ellipsoidal $\mathscr{G}$ (Proposition 1 ). In Section 3 we introduce the minimax observable sub-

1 DAE is said to be non-causal if the corresponding initialvalue problem has more than one solution.

2 Note that the dynamic programming was previously applied to causal DAEs in [6] in order to construct a regulator in LQ-control problems with DAE constraints. space and index of non-causality for DAEs in discrete time (Definition 2) and we derive the minimax estimator (Theorem 1). Also we discuss connections to $H_{2} / H_{\infty}$ framework (Corollary 1) and present an example.

Notation. Linear mappings: $\langle\cdot, \cdot\rangle$ denotes the inner product; $\mathscr{L}\left(\mathcal{H}_{1}, \mathcal{H}_{2}\right)$ denotes the space of all bounded linear mappings from $\mathcal{H}_{1}$ to $\mathcal{H}_{2}, \mathscr{L}(\mathcal{H}):=\mathscr{L}(\mathcal{H}, \mathcal{H})$; $\mathbb{1}_{\mathcal{H}}$ is the identity mapping in $\mathcal{H} ; \mathscr{D}(L), R(L), N(L)$ denote, respectively, the domain, range, and null-space of a linear mapping $L: \mathscr{D}(L) \mapsto R(L) ; L^{*}: \mathcal{H} \rightarrow \mathcal{H}$ is the adjoint of $L ; F^{\prime}$ denotes the transpose of $F ; F^{+}$ is the pseudoinverse of $F$; $E$ is the identity matrix; $\operatorname{diag}\left(A_{1} \ldots A_{n}\right)$ denotes a diagonal matrix with $A_{i}$, $i=\overline{1, n}$ on its diagonal; $\left\{x_{s}\right\}_{1}^{n}:=\left(x_{1}, \ldots, x_{n}\right)$ is an element of $\mathcal{H}_{1} \times \cdots \times \mathcal{H}_{n}, 0_{m n} \in \mathbb{R}^{m \times n}$ denotes the $m \times n$-zero matrix.

Functionals: $I_{1}(x):=\left\langle Q_{1} L x, L x\right\rangle+\left\langle Q_{2} H x, H x\right\rangle$, $I(x):=\left\langle Q_{1} L x, L x\right\rangle+\left\langle Q_{2}(y-H x), y-H x\right\rangle$ with positive definite self-adjoint $Q_{1} \in \mathscr{L}(\mathcal{F})$ and $Q_{2} \in \mathscr{L}(\mathcal{Y})$; $c(G, x):=\sup \{\langle x, y\rangle, y \in G\}$ is a support function of $G$; $\gamma_{ \pm}:=\frac{1}{2}(c(\mathscr{G}(y), \ell) \pm c(\mathscr{G}(y),-\ell)) ;\|x\|_{S}^{2}=\langle S x, x\rangle$.

Sets: $\mathscr{G}^{\beta}(0):=\left\{x: I_{1}(x) \leq \beta\right\}, \mathscr{G}(0):=\left\{x: I_{1}(x) \leq 1\right\}$ $\operatorname{dom} f:=\{x: f(x)<\infty\}$ is an effective domain of $f$ $\operatorname{Argmin}_{x} f:=\left\{x: f(x)=\min _{x} f\right\}$ is the set of global minima of $f ; \bar{G}$ is the closure of a set $G$.

\section{Linear minimax estimation problem in a Hilbert space}

Let vector $y$ be observed in the form of

$$
y=H \varphi+\eta
$$

where $\varphi$ obeys the equation

$$
L \varphi=f
$$

We assume that $L: \mathscr{D}(L) \mapsto \mathcal{F}$ is a closed linear operator $[4$, p.63], $\mathscr{D}(L) \subset \mathcal{H}$ is a linear set, $\overline{\mathscr{D}(L)}=\mathcal{H}$, $H \in \mathscr{L}(\mathcal{H}, \mathcal{Y})$. Also we assume that $(f, \eta)$ is an unknown element of a convex bounded closed set $\mathscr{G} \subset \mathcal{F} \times \mathcal{Y}$, $\mathcal{H}, \mathcal{F}, \mathcal{Y}$ are Hilbert spaces.

Definition 1 Let $\mathcal{L}:=\{\ell \in \mathcal{H}: \hat{\rho}(\ell)<\infty\}$ with

$$
\hat{\rho}(\ell):=\inf _{\varphi \in \mathscr{G}(y)} \rho(\ell, \varphi), \quad \rho(\ell, \varphi):=\sup _{\psi \in \mathscr{G}(y)}|\langle\ell, \varphi-\psi\rangle|
$$

The set $\mathcal{L}$ is called a minimax observable subspace for the pair $(L, H)$. A vector $\hat{\varphi} \in \mathscr{G}(y)$ is called a minimax a posteriori estimation in the direction ${ }^{3} \ell$ ( $\ell$-estimation) if $\rho(\ell, \hat{\varphi})=\hat{\rho}(\ell)$. The number $\hat{\rho}(\ell)$ is called a minimax a posteriori error in the direction $\ell$ ( $\ell$-error).

3 The subspace $l:=\{\alpha \ell: \alpha \in \mathbb{R}\}$, assigned with $\ell \in \mathcal{H}$, defines some direction in $\mathcal{H}$. To estimate $\varphi$ in the direction $\ell$ means to estimate the projection $\langle\ell, \varphi\rangle \ell$ of $\varphi$ onto $l$. 
Our aim here $i s$, given $y$, to construct the $\ell$-estimation $\hat{\varphi}$ of the state $\varphi, \ell$-error $\hat{\rho}(\ell)$ and minimax observable subspace $\mathcal{L}$, provided $\ell \in \mathcal{L}$. Note that $\hat{\rho}(\ell)=+\infty$ if $\ell \notin \mathcal{L}$ so that any $\psi \in \mathcal{H}$ is a $\ell$-estimation by Definition 1 .

Proposition 1 Let $\mathscr{G}$ be a convex closed bounded subset of $\mathcal{F} \times \mathcal{Y}$. Then $\ell \in \mathcal{L}$ iff $\ell,-\ell \in \operatorname{dom} c(\mathscr{G}(y), \cdot)$. If $\ell \in \mathcal{L}$ then the $\ell$-estimation $\hat{\varphi}$ along with $\ell$-error $\hat{\rho}(\ell)$ obey

$$
\langle\ell, \hat{\varphi}\rangle=\gamma_{-}, \hat{\rho}(\ell)=\gamma_{+}
$$

Define $T: \mathscr{D}(T) \rightarrow \mathcal{H}$ by the rule $T(z, u):=L^{*} z+H^{*} u$ with $\mathscr{D}(T):=\mathscr{D}\left(L^{*}\right) \times \mathcal{Y}$ and let

$$
\mathscr{G}=\left\{(f, \eta):\left\langle Q_{1} f, f\right\rangle+\left\langle Q_{2} \eta, \eta\right\rangle \leq 1\right\}
$$

If $R(T)=\overline{R(T)}$ then $\hat{x} \in \operatorname{Argmin}_{x} I$ is the l-estimation, $\mathcal{L}=\operatorname{dom} c(\mathscr{G}(0), \cdot)=R(T)$ and

$$
\hat{\rho}(\ell)=(1-I(\hat{x}))^{\frac{1}{2}} c(\mathscr{G}(0), \ell) .
$$

The worst-case estimation error for any direction is

$$
\begin{aligned}
& \sup _{x \in \mathscr{G}(y)}\|\hat{x}-x\|=\inf _{\varphi \in \mathscr{G}(y)} \sup _{x \in \mathscr{G}(y)}\|\varphi-x\|= \\
& (1-I(\hat{x}))^{\frac{1}{2}} \sup _{\|\ell\|=1} c(\mathscr{G}(0), \ell)=\sup _{\|\ell\|=1} \hat{\rho}(\ell)<+\infty
\end{aligned}
$$

If $\mathcal{L}=\mathcal{H}$ then $(6)$ is finite.

PROOF. Let $\ell(\mathscr{G}(y))=\{\langle\ell, \psi\rangle, \psi \in \mathscr{G}(y)\}$. Since $\mathscr{G}(y)$ is convex (due to convexity of $\mathscr{D}(L)$ and $\mathscr{G}$ ) and $x \mapsto$ $\langle\ell, x\rangle$ is continuous, it follows that $\overline{\ell(\mathscr{G}(y))}$ is connected. Noting that $\inf _{\mathscr{G}(y)}\langle\ell, \psi\rangle=-c(\mathscr{G}(y),-\ell)$, we see

$$
\overline{\ell(\mathscr{G}(y))}=[-c(\mathscr{G}(y),-\ell), c(\mathscr{G}(y), \ell)] \subset \mathbb{R}^{1}
$$

Thus $\rho(\ell, \varphi)=+\infty$ if $\ell,-\ell \notin \operatorname{dom} c(\mathscr{G}(y), \cdot)$. Otherwise $\overline{\ell(\mathscr{G}(y))}$ is bounded, implying $\ell \in \mathcal{L}$. Hence, $\ell \in \mathcal{L}$ iff $\ell,-\ell \in \operatorname{dom} c(\mathscr{G}(y), \cdot)$.

Let $\ell \in \mathcal{L}, \varphi \in \mathscr{G}(y)$. Since $\overline{\ell(\mathscr{G}(y))}$ is connected, there exists $\varphi_{*} \in \mathscr{G}(y)$ so that $\left\langle\ell, \varphi_{*}\right\rangle=\gamma_{-}$is the central point of $\overline{\ell(\mathscr{G}(y))}$. The worst-case distance $\rho(\ell, \varphi)$ is equal to the sum of the distance $\left|\left\langle\ell, \varphi-\varphi^{*}\right\rangle\right|$ between $\langle\ell, \varphi\rangle$ and the central point $\gamma_{-}$and the distance $\gamma_{+}$between one of the boundary points of $\overline{\ell(\mathscr{G}(y))}$ and $\gamma_{-}$. Therefore, $\gamma_{-}$ has the minimal worst-case distance $\gamma_{+}$. Hence, $\varphi_{*}=\hat{\varphi}$ due to Definition 1, which implies (4).

We proceed with the ellipsoidal $\mathscr{G}$. Let $Q_{1}=\mathbb{1}_{\mathcal{F}}, Q_{2}=$ $\mathbb{1}_{\mathcal{Y}}$ for a simplicity. Due to $[11, \operatorname{Sec} 5 . \S 3] R(T)=\overline{R(T)}$ implies $R\left(T^{*}\right)=\overline{R\left(T^{*}\right)}$. Thus [4, p.14,Cor.1.4.3], there exists $\hat{x} \in \mathscr{D}(L)$ so that $T^{*} \hat{x}$ is the projection of $(0, y)$ onto $R\left(T^{*}\right)=\{(L x, H x), x \in \mathscr{D}(L)\}$, implying $\hat{x} \in$ $\operatorname{Argmin}_{x} I$, and

$$
\langle y-H \hat{x}, H x\rangle=\langle L \hat{x}, L x\rangle, \forall x \in \mathscr{D}(L)
$$

Noting this, one easily derives ${ }^{4} I(\hat{x}-x)=I_{1}(x)+I(\hat{x})$ for all $x \in \mathscr{D}(L)$. Having it in mind and noting that $\mathscr{G}(y)=\{\varphi: I(\varphi) \leqslant 1\}$ and $\hat{x} \in \mathscr{G}(y)$, one derives

$$
\hat{x}+\mathscr{G}^{\beta}(0)=\mathscr{G}(y)
$$

where $\beta:=1-I(\hat{x}) .(7)$ implies $[18, \mathrm{p} .113]$

$$
c(\mathscr{G}(y), \ell)=\langle\ell, \hat{x}\rangle+c\left(\mathscr{G}^{\beta}(0), \ell\right), \forall \ell \in \mathcal{H}
$$

The definition of $\gamma_{-},(8)$ and $c\left(\mathscr{G}^{\beta}(0), \ell\right)=c\left(\mathscr{G}^{\beta}(0),-\ell\right)$ imply $\gamma_{-}=\langle\ell, \hat{x}\rangle$. Due to $(4), \hat{x}$ is the $\ell$-estimation.

Let us prove (5). If $x \in \mathscr{G}^{\beta}(0)$ then $I_{1}\left(\beta^{-\frac{1}{2}} x\right) \leq 1$. Thus

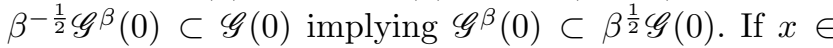
$\mathscr{G}(0)$ then $I_{1}\left(\beta^{\frac{1}{2}} x\right)=\beta I_{1}(x) \leq \beta \Rightarrow \beta^{\frac{1}{2}} \mathscr{G}(0) \subset \mathscr{G}^{\beta}(0)$. Therefore

$$
\mathscr{G}^{\beta}(0)=\beta^{\frac{1}{2}} \mathscr{G}(0) \Rightarrow c\left(\mathscr{G}^{\beta}(0), \ell\right)=\beta^{\frac{1}{2}} c(\mathscr{G}(0), \ell)
$$

Now (4) and (8) imply (5). Hence, $\mathcal{L}=\operatorname{dom} c(\mathscr{G}(0), \cdot)$ due to Definition 1.

Let us prove $R(T)=\mathcal{L}$. If $\ell \in R(T)$ then $\ell=L^{*} z+H^{*} u$ for some $z \in \mathscr{D}\left(L^{*}\right), u \in \mathcal{Y}$ and we get $\forall \varphi \in \mathscr{G}(0)$ by Cauchy inequality $[4$, p.4]

$$
\langle\ell, \varphi\rangle=\langle z, L \varphi\rangle+\langle u, H \varphi\rangle \leq\|z\|^{2}+\|u\|^{2}<+\infty
$$

so that $R(T) \subset \operatorname{dom} c(\mathscr{G}(0), \cdot)=\mathcal{L}$. If $\ell \notin R(T)$ then $\langle\ell, x\rangle>0$ for some $x \in N\left(T^{*}\right)$ as $\mathcal{H}=\overline{R(T)} \oplus N\left(T^{*}\right)$. Noting that $\mathscr{G}(0)=\left\{x:\left\|T^{*} x\right\|^{2} \leq 1\right\}$ we derive $c(\mathscr{G}(0), \ell) \geq \sup \left\{\langle\ell, x\rangle: T^{*} x=0\right\}=+\infty$. Hence, $\mathcal{L} \subset R(T)$.

Let us prove (6). Set $\hat{\alpha}:=\inf _{\varphi \in \mathscr{G}(y)} \sup _{x \in \mathscr{G}(y)}\|\varphi-x\|$. Using Definition 1 , one derives ${ }^{5}$

$$
\begin{aligned}
& \hat{\alpha}=\inf _{\varphi \in \mathscr{G}(y)} \sup _{x \in \mathscr{G}(y)} \sup _{\|\ell\|=1}|\langle\ell, \varphi-x\rangle| \geq \\
& \sup _{\|\ell\|=1} \inf _{\varphi \in \mathscr{G}(y)} \sup _{x \in \mathscr{G}(y)}|\langle\ell, \varphi-x\rangle|=\sup _{\|\ell\|=1} \hat{\rho}(\ell)= \\
& \sup _{\|\ell\|=1} \sup _{x \in \mathscr{G}(y)}|\langle\ell, \hat{x}-x\rangle|=\sup _{x \in \mathscr{G}(y)}\|\hat{x}-x\| \geq \hat{\alpha}
\end{aligned}
$$

Now assume $\mathcal{L}=\mathcal{H}$. Since $\operatorname{dom} c(\mathscr{G}(0), \cdot)=\mathcal{L}$, it follows $c(\mathscr{G}(0), \cdot)$ is finite in $\mathcal{H}$ and therefore $[9, \S 2.3]$ continuous. As a consequence, (6) is finite.

${ }^{4} I(\hat{x}-x)=I_{1}(x)+I(\hat{x})-2\langle L \hat{x}, L x\rangle+2\langle y-H \hat{x}, H x\rangle$

5 Note [4, p.42] that $\|\varphi\|=\sup _{\|\ell\|=1}\langle\ell, \varphi\rangle$ and [4, p.55] $\inf _{x} \sup _{y} F(x, y) \geq \sup _{y} \inf _{x} F(x, y)$ for convex-concave $F$. 


\section{$3 \ell$-estimation for non-causal DAEs}

Consider the model

$$
\begin{aligned}
& F_{k+1} x_{k+1}-C_{k} x_{k}=f_{k+1}, F_{0} x_{0}=f_{0}, \\
& y_{k}=H_{k} x_{k}+g_{k}, k=0,1, \ldots
\end{aligned}
$$

where $F_{k}, C_{k} \in \mathbb{R}^{m \times n}, H_{k} \in \mathbb{R}^{p \times n}, x_{k} \in \mathbb{R}^{n}$ is a state, $f_{k} \in \mathbb{R}^{m}$ is an input and $y_{k}, g_{k} \in \mathbb{R}^{p}$ represent an output and the output's noise respectively. In what follows we assume that an initial state $x_{0}$ belongs to the affine set $\left\{x: F_{0} x=f_{0}\right\}$. We define $\xi_{\tau}=\left(\left\{f_{s}\right\}_{0}^{\tau},\left\{g_{s}\right\}_{0}^{\tau}\right)$ and assume

$$
\xi_{\tau} \in \mathscr{G}=\left\{\xi_{\tau}: \sum_{0}^{\tau}\left\langle S_{i} f_{i}, f_{i}\right\rangle+\left\langle R_{i} g_{i}, g_{i}\right\rangle \leqslant 1\right\}
$$

where $S_{k} \in \mathbb{R}^{m \times m}$ and $R_{k} \in \mathbb{R}^{p \times p}$ are positive definite self-adjoint matrices.

Suppose we observe $y_{1}^{*}, \ldots, y_{\tau}^{*}$, provided that $y_{k}^{*}$ is derived from (11) with $g_{k}=g_{k}^{*}$ and $x_{k}=x_{k}^{*}$, which obeys (10) with $f_{k}=f_{k}^{*}$, and $\left(\left\{f_{s}^{*}\right\}_{0}^{\tau},\left\{g_{s}^{*}\right\}_{0}^{\tau}\right) \in \mathscr{G}$. Denote by $X(\tau)$ the set of all possible states $x_{\tau}$ of (10) consistent with measurements $y_{1}^{*}, \ldots, y_{\tau}^{*}$ and uncertainty description (12).

Definition 2 We say that $\bar{x}_{\tau}$ is a -estimation of the state $x_{\tau}^{*}$ in the direction $\ell \in \mathbb{R}^{n}$ iff

$\sup _{x \in X(\tau)}\left|\left\langle\ell, x-\bar{x}_{\tau}\right\rangle\right|=\hat{\rho}(\ell, \tau):=\inf _{z \in X(\tau)} \sup _{x \in X(\tau)}|\langle\ell, x-z\rangle|$

$\hat{\rho}(\ell, \tau)$ is said to be an $\ell$-error. A minimax observable subspace at the instant $\tau$ for the model (10)-(11) is defined by $\mathcal{L}(\tau)=\{\ell: \hat{\rho}(\ell, \tau)<\infty\} . I_{\tau}:=n-\operatorname{dim} \mathcal{L}(\tau)$ is called an index of non-causality of the model (10)-(11).

Theorem 1 Define $\hat{\beta}_{\tau}:=1-\alpha_{\tau}+\left\langle P_{\tau} \hat{x}_{\tau}, \hat{x}_{\tau}\right\rangle$ and $\hat{x}_{\tau}=$ $P_{\tau}^{+} r_{\tau}$ with $r_{0}=H_{0}^{\prime} R_{0} y_{0}, \alpha_{0}=\left\langle R_{0} y_{0}, y_{0}\right\rangle$,

$P_{k}=H_{k}^{\prime} R_{k} H_{k}+F_{k}^{\prime}\left[S_{k-1}-S_{k-1} C_{k-1} B_{k-1}^{+} C_{k-1}^{\prime} S_{k-1}\right] F_{k}$,

$P_{0}=F_{0}^{\prime} S_{0} F_{0}+H_{0}^{\prime} R_{0} H_{0}, B_{k}=P_{k}+C_{k}^{\prime} S_{k} C_{k}$,

$\alpha_{k}=\alpha_{k-1}+\left\langle R_{k} y_{k}, y_{k}\right\rangle-\left\langle B_{k-1}^{+} r_{k-1}, r_{k-1}\right\rangle$,

$r_{k}=F_{k}^{\prime} S_{k-1} C_{k-1} B_{k-1}^{+} r_{k-1}+H_{k}^{\prime} R_{k} y_{k}$,

Then $\hat{x}_{\tau}$ is the $\ell$-estimation of $x_{\tau}^{*}, \mathcal{L}(\tau)=\left\{\ell: P_{\tau}^{+} P_{\tau} \ell=\right.$ $\ell\}, \hat{\rho}(\ell, \tau)=\hat{\beta}_{\tau}^{\frac{1}{2}}\left\langle P_{\tau}^{+} \ell, \ell\right\rangle^{\frac{1}{2}}$ and $^{6}$

$$
X(\tau)=\hat{x}_{\tau}+\hat{\beta}_{\tau}^{\frac{1}{2}} \tilde{X}(\tau), \tilde{X}(\tau):=\left\{x:\left\langle P_{\tau} x, x\right\rangle \leq 1\right\}
$$

\footnotetext{
6 Note that $\tau \mapsto X(\tau)$ represents the a posteriori set-valued observer [20]
}

PROOF. In order to apply Proposition 1, we rewrite (10)(11) in the operator form: set $\mathcal{H}=\left(\mathbb{R}^{n}\right)^{\tau+1}, \mathcal{Y}=$ $\left(\mathbb{R}^{p}\right)^{\tau+1}, \mathcal{F}=\left(\mathbb{R}^{m}\right)^{\tau+1}$ and $\varphi^{*}=\left\{x_{s}^{*}\right\}_{0}^{\tau}, y^{*}=\left\{y_{s}^{*}\right\}_{0}^{\tau}$, $f^{*}=\left\{f_{s}^{*}\right\}_{0}^{\tau}, \eta^{*}=\left\{g_{s}^{*}\right\}_{0}^{\tau}$,

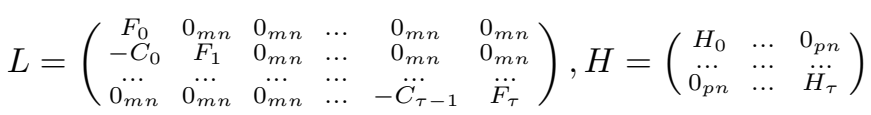

Define $\mathcal{P}_{\tau}=\left(0_{n n}, \ldots, 0_{n n}, E\right)$ and rewrite (12) with $Q_{1}=\operatorname{diag}\left(S_{0}, \ldots, S_{\tau}\right), Q_{2}=\operatorname{diag}\left(R_{0}, \ldots, R_{\tau}\right)$ as

$$
\xi_{\tau}=(f, \eta) \in \mathscr{G}=\left\{(f, \eta):\left\langle Q_{1} f, f\right\rangle+\left\langle Q_{2} \eta, \eta\right\rangle \leq 1\right\} .
$$

It is clear that $y^{*}, H, \eta^{*}, L, f^{*}, \varphi^{*}$, defined as above, satisfy (2)-(3) and $\left(f^{*}, \eta^{*}\right) \in \mathscr{G}$. Let $\mathscr{G}\left(y^{*}\right)$ denote the a posteriori set generated by $y^{*}$. Then $X(\tau)=\mathcal{P}_{\tau}\left(\mathscr{G}\left(y^{*}\right)\right)$ by definition. Thus

$$
\hat{\rho}(\ell, \tau)=\inf _{\varphi \in \mathscr{G}\left(y^{*}\right)} \sup _{\psi \in \mathscr{G}\left(y^{*}\right)} \mid\left\langle\ell, \mathcal{P}_{\tau}(\varphi-\psi)\right|=\hat{\rho}(l)
$$

with $l=\mathcal{P}_{\tau}^{\prime} \ell$. Hence, $\bar{x}_{\tau}=\mathcal{P}_{\tau} \hat{\varphi}$, where $\hat{\varphi}$ is the $l$-minimax estimation of the state $\varphi^{*}$ of $(3)$ in the sense of Definition 1. Proposition 1 implies $\hat{\varphi}=\hat{x}$ and $\hat{\rho}(l)=\beta^{\frac{1}{2}} c(\mathscr{G}(0), l)$. Let us prove (13). (7) and (9) imply $\mathcal{P}_{\tau}\left(\mathscr{G}\left(y^{*}\right)\right)=\mathcal{P}_{\tau} \hat{x}+\beta^{\frac{1}{2}} \mathcal{P}_{\tau}(\mathscr{G}(0))$. Therefore, $X(\tau)=\mathcal{P}_{\tau} \hat{x}+\beta^{\frac{1}{2}} \mathcal{P}_{\tau}(\mathscr{G}(0))$. Now, let us prove $\hat{x}_{\tau}=\mathcal{P}_{\tau} \hat{x}$ by the direct calculation. Define

$$
V_{\tau}\left(x_{0}, \ldots, x_{\tau}\right):=\Phi\left(x_{0}\right)+\sum_{s=0}^{\tau-1} \Phi_{s}\left(x_{s}, x_{s+1}\right)
$$

with $\Phi_{k}(x, p):=\left\|F_{k+1} x-C_{k} p\right\|_{S_{k+1}}^{2}+\| y_{k+1}-$ $H_{k+1} x\left\|_{R_{k+1}}^{2}, \Phi(x):=\right\| F_{0} x\left\|_{S_{0}}^{2}+\right\| y_{0}-H_{0} x \|_{R_{0}}^{2}$, and

$$
\mathcal{B}_{\tau}(p):=\min _{x_{0} \ldots x_{\tau-1}} V_{\tau}\left(x_{0} \ldots x_{\tau-1}, p\right), \mathcal{B}_{0}(p):=\Phi(p)
$$

Lemma 1. Let $p \in \mathbb{R}^{n}$. There exists $\left(\tilde{x}_{1} \ldots \tilde{x}_{\tau-1}\right) \in$ $\left(R^{n}\right)^{\tau-1}$ so that $V_{\tau}\left(\tilde{x}_{1} \ldots \tilde{x}_{\tau-1}, p\right)=\mathcal{B}_{\tau}(p)$ and

$$
\mathcal{B}_{k}(p)=\left\langle P_{k} p, p\right\rangle-2\left\langle r_{k}, p\right\rangle+\alpha_{k}, P_{k} \geq 0
$$

Lemma 1 implies $\mathcal{B}_{\tau}$ is a quadratic and non-negative function. Therefore $\operatorname{Argmin} \mathcal{B}_{\tau}=\left\{x: P_{\tau} x=r_{\tau}\right\} \neq \varnothing$. This and $I(\varphi)=V_{\tau}\left(x_{0}, \ldots, x_{\tau}\right)$ imply

$$
\mathcal{B}_{\tau}\left(\hat{x}_{\tau}\right)=\min \mathcal{B}_{\tau}=\min V_{\tau}=\min I
$$

Defining $\hat{x}:=\left(\tilde{x}_{1} \ldots \tilde{x}_{\tau-1}, \hat{x}_{\tau}\right)$ with $\tilde{x}_{k}$ taken as in Lemma 1 for $p=\hat{x}_{\tau}$, we obtain $I(\hat{x})=\min I$ and $\hat{x}_{\tau}=\mathcal{P}_{\tau} \hat{x}$. Therefore, $\hat{x}_{\tau}$ is a $\ell$-estimation.

We note $\min I=\mathcal{B}_{\tau}\left(\hat{x}_{\tau}\right)=1-\hat{\beta}_{\tau}$. Thus $\beta=\hat{\beta}_{\tau}$ by definition.

Let us prove $\mathcal{P}_{\tau}(\mathscr{G}(0))=\tilde{X}(\tau)$. Since $\mathscr{G}(0)$ does not depend on $y^{*}$ and $\mathscr{G}(0)=\mathscr{G}\left(y^{*}\right)$ provided $y^{*}=0$, 
we can calculate $\mathcal{P}_{\tau}(\mathscr{G}(0))$ assuming $y^{*}=0$. In this case $I=I_{1}=V_{\tau}, \hat{x}_{\tau}=0$ and $\mathcal{B}_{\tau}(x)=\left\langle P_{\tau} x, x\right\rangle$ so that $x \in \tilde{X}(\tau) \Leftrightarrow \mathcal{B}_{\tau}(x) \leq 1$. If $x \in \mathcal{P}_{\tau}(\mathscr{G}(0))$ then, by definition, there exist $x_{1} \ldots x_{\tau-1}$ so that $V_{\tau}\left(x_{1} \ldots x_{\tau-1}, x\right) \leq 1$, implying $\mathcal{B}_{\tau}(x) \leq 1$. Now, let $\mathcal{B}_{\tau}(x) \leq 1$. Then $V_{\tau}\left(\tilde{x}_{1} \ldots \tilde{x}_{\tau-1}, x\right)=\mathcal{B}_{\tau}(x)$ due to Lemma 1 and thus $x \in \mathcal{P}_{\tau}(\mathscr{G}(0))$ by definition.

Formulae (5), (14) and $\mathcal{P}_{\tau}(\mathscr{G}(0))=\tilde{X}(\tau)$ imply $\hat{\rho}(\ell, \tau)=\hat{\beta}_{\tau}^{\frac{1}{2}} c(\tilde{X}(\tau), \ell)=\hat{\beta}_{\tau}^{\frac{1}{2}}\left\langle P_{\tau}^{+} \ell, \ell\right\rangle^{\frac{1}{2}}$ for $\ell \in \mathcal{L}(\tau)$ and $\mathcal{L}(\tau)=\operatorname{dom} c(\tilde{X}(\tau), \cdot)=\left\{\ell: P_{\tau}^{+} P_{\tau} \ell=\ell\right\}$. Details of calculation of $c(\tilde{X}(\tau), \cdot)$ are given in [18, p.108]. This completes the proof.

Proof of Lemma 1. We shall apply the dynamic programming [2]. Since $V_{\tau}$ is additive, it follows that

$$
\mathcal{B}_{\tau}(p)=\min _{x_{\tau-1}}\left\{\Phi_{\tau-1}\left(x_{\tau-1}, p\right)+\mathcal{B}_{\tau-1}\left(x_{\tau-1}\right)\right\}
$$

$V_{\tau}$ is convex and non-negative by definition. Thus $\mathcal{B}_{\tau}$ is non-negative and convex for any $\tau \in \mathbb{N}$. Convexity is implied by the definition of $\mathcal{B}_{\tau}$ as for any convex function $(x, y) \mapsto f(x, y)$ the function $y \mapsto \min _{x} f(x, y)$ is convex [18, p.38].

Let us prove (15) by induction. (15) holds for $\mathcal{B}_{0}$ and $P_{0}$. We shall derive $(15)$ for $\mathcal{B}_{k+1}, P_{k+1}$, assuming it holds for $\mathcal{B}_{k}, P_{k}$. Define

$$
\Xi_{k}(x, p):=\Phi_{k}(x, p)+\left\langle P_{k} x, x\right\rangle-2\left\langle r_{k}, x\right\rangle+\alpha_{k}
$$

Then $\mathcal{B}_{k+1}(p)=\min _{x_{k}} \Xi_{k}\left(x_{k}, p\right)$ due to (16). Combining $P_{k} \geq 0$ with definition of $\Phi_{k}$, we derive ${ }^{7}$ $x \mapsto \Xi_{k}(x, p)$ is a convex quadratic function for any $p$. This and $\Xi_{k}\left(x_{k}, p\right) \geq B_{k+1}(p) \geq 0$ imply [18, p.268] $\operatorname{Argmin}_{x} \Xi_{k}(x, p) \neq \varnothing$. On the other hand [18, T.27.4] $x \in \operatorname{Argmin}_{x} \Xi_{k}(x, p)$ iff $\nabla_{x} \Xi_{k}(x, p)=0$. Finally, we $\operatorname{obtain}_{\operatorname{Argmin}} \Xi_{k}(x, p) \neq \varnothing$ and

$$
\operatorname{Argmin}_{x} \Xi_{k}(x, p)=\left\{x: B_{k} x=C_{k}^{\prime} S_{k} F_{k+1} p+r_{k}\right\}
$$

If we set $q_{k}=B_{k}^{+}\left(C_{k}^{\prime} S_{k} F_{k+1} p+r_{k}\right)$ then $q_{k} \in$ $\operatorname{Argmin}_{x} \Xi_{k}(x, p)$ due to [1]. Now, it is sufficient to calculate $\Xi_{k}\left(q_{k}, p\right)$ in order to see that (15) holds for $\mathcal{B}_{k+1}$ and $P_{k+1}$. Assertion $P_{k+1} \geq 0$ holds since $\mathcal{B}_{k+1}$ is convex. To conclude the proof, let us define $\tilde{x}_{\tau}=p$ and $\tilde{x}_{k} \in \operatorname{Argmin}_{x} \Xi_{k}\left(x, \tilde{x}_{k+1}\right), k=\overline{1, \tau-1}$. Then $V_{\tau}\left(\tilde{x}_{1} \ldots \tilde{x}_{\tau-1}, p\right)=\mathcal{B}_{\tau}(p)$ due to $(16)-(18)$.

3.1. Example. Consider a system $p_{k+1}=A_{k} p_{k}+$ $v_{k}, p_{0}=v$ and assume $y_{k}=\tilde{H}_{k} p_{k}+g_{k}$ provided $p_{k} \in \mathbb{R}^{n}$ and $\left(v, g_{0}, \ldots, g_{\tau}\right)$ belong to some ellipsoid. Now, given $y_{1}^{*}, \ldots, y_{\tau}^{*}$, one needs to build the worst-case estimation of $p_{\tau}^{*}$. We cannot apply directly standard minimax

${ }^{7} x \mapsto\langle A x, x\rangle-2\langle x, q\rangle+c$ is convex iff $A$ is a symmetric non-negative matrix. framework $[16,7,5]$ in this case as we do not have any information about the bounding set for $\left(v_{0}, \ldots, v_{\tau}\right)$. Instead, we apply the approach ${ }^{8}$, proposed above. Define $F_{k}:=(E, 0), C_{k}:=\left(A_{k}, E\right), H_{k}:=\left(\tilde{H}_{k}, 0\right)$ and $x_{k}^{*}:=\left(p_{k}^{*}, v_{k}^{*}\right)$. Then $x_{k}^{*}$ verifies $(10)-(11)$ with $f_{0}=v$, $f_{k}=0$ and $g_{k}=g_{k}^{*}, k=\overline{1, \tau}$. Therefore, the original problem may be reformulated as: given $y_{1}^{*}, \ldots, y_{\tau}^{*}$, one needs to build the $l=(\ell, 0)$-minimax estimation of $x_{\tau}^{*}$. Of course, the estimation of $x_{\tau}$ in the direction $l=(0, \ell)$ has an infinite minimax error for any $\ell$. But this is natural as $\left\langle l, x_{\tau}\right\rangle=\left\langle v_{\tau}, \ell\right\rangle$ and $\left(v_{1}, \ldots, v_{\tau}\right)$ is unknown.

In what follows we present a numerical example. Let $p_{k} \in \mathbb{R}^{2}, \tilde{H}_{k}=\left(\begin{array}{ll}1 & 0\end{array}\right), A_{k}=\left(\begin{array}{cc}\frac{1}{10} & -\frac{1}{5} \\ \frac{7}{25} & -\frac{1}{10}\end{array}\right)$ and $v_{k}=\left(-\frac{k \sin (k)}{10}, \frac{k \sin (k)}{10}\right)$. We have generated $p_{k}^{*}$ with $v=\left(\frac{1}{10}, \frac{1}{10}\right)$ and $y_{k}^{*}$ with $g_{k}=\frac{2 \sin (k)}{k+1}, R_{k}=\frac{k}{k+1}$, $S_{k}=\operatorname{diag}(1,1), k=\overline{0,50}$. The results are displayed on Fig. 1.

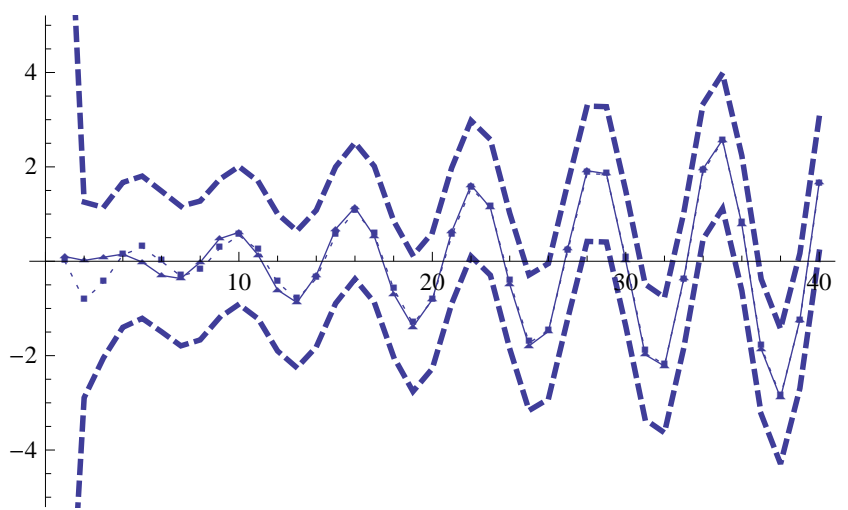

Fig. 1. The dashed line corresponds to the real values of $\left\langle\ell, p_{k}^{*}\right\rangle$ with $\ell=(0,1), k=\overline{1,40}$; the solid line corresponds to the $\ell$-estimation $\left\langle\ell, \hat{x}_{k}\right\rangle$; the bold dashed lines represent dynamics of the boundary points of the segment $\ell(\tilde{X}(\tau))$. Note that the trajectory of the estimation is centered with respect to "the bounds" - bold dashed lines.

3.2. Minimax estimator and $H_{2} / H_{\infty}$ filters. In [5] a connection between set-membership state estimation and $H_{\infty}$ approach is described for linear causal DAEs. The authors note that the notion of informational state $(X(\tau)$ in our notation) is shown to be intrinsic for both approaches: mathematical relations between informational states of $H_{\infty}$ and set-membership state estimation are described in [5, Lemma 6.2.]. Comparisons of set-membership estimators with $\mathrm{H}_{2} / \mathrm{H}_{\infty}$ filters for linear DAEs are presented in [19], provided $F_{k} \equiv E$. Let us consider connections to $\mathrm{H}_{2}$-filters in details. In [10] the authors derive the Kalman's recursion to DAE from a deterministic least square fitting problem. Assuming $\operatorname{rank}\left[\begin{array}{c}F_{k} \\ H_{k}\end{array}\right] \equiv n$, they prove that the optimal estimation

\footnotetext{
8 Since $\operatorname{rank}\left[\begin{array}{c}F_{k} \\ H_{k}\end{array}\right]<2 n$, it follows that results of [10] are not applicable.
} 
$\hat{x}_{i \mid k}$ can be found from

$$
\begin{aligned}
& \hat{x}_{k \mid k}=P_{k \mid k} F_{k}^{\prime} A_{k-1} C_{k-1} \hat{x}_{k-1 \mid k-1}+P_{k \mid k} H_{k}^{\prime} R_{k} y_{k}, \\
& \hat{x}_{0 \mid 0}=P_{0 \mid 0} H_{0}^{\prime} R_{0} y_{0}, A_{k}^{-1}=S_{k}^{-1}+C_{k} P_{k \mid k} C_{k}^{\prime} \\
& P_{k \mid k}^{-1}=F_{k}^{\prime} A_{k-1} F_{k}+H_{k}^{\prime} R_{k} H_{k}, P_{0 \mid 0}^{-1}=F_{0}^{\prime} S F_{0}+H_{0}^{\prime} R_{0} H_{0}
\end{aligned}
$$

Corollary 1 Let $r_{0}=H_{0}^{\prime} R_{0} y_{0}$. If $\operatorname{rank}\left[\begin{array}{c}F_{k} \\ H_{k}\end{array}\right] \equiv n$ then $I_{k}=0$ and $P_{k}^{+} r_{k}=\hat{x}_{k \mid k}$.

PROOF. Let us set $R_{k}=E, S=E, S_{k}=E$ for simplicity. The proof is by induction on $k$. For $k=0, P_{0 \mid 0}=$ $P_{0}^{-1}$. The induction hypothesis is $P_{k-1 \mid k-1}=P_{k-1}^{-1}$. Suppose $A \in \mathbb{R}^{m \times n}, B \in \mathbb{R}^{n \times n}, B=B^{\prime}>0$; then

$$
A\left(A^{\prime} A+B^{-1}\right)^{-1}=\left(E+A B A^{\prime}\right)^{-1} A B
$$

Using (19) we get $A B A^{\prime}=\left[E+A B A^{\prime}\right] A\left[A^{\prime} A+\right.$ $\left.B^{-1}\right]^{-1} A^{\prime}$. Combining this with the induction assumption we get $E+C_{k-1} P_{k-1 \mid k-1} C_{k-1}^{\prime}=E+[E+$ $\left.C_{k-1} P_{k-1 \mid k-1} C_{k-1}^{\prime}\right] C_{k-1}\left[P_{k-1}+C_{k-1}^{\prime} C_{k-1}\right]^{-1} C_{k-1}^{\prime}$. By simple calculation it follows from the previous equality that $E-C_{k-1}\left(P_{k-1}+C_{k-1}^{\prime} C_{k-1}\right)^{-1} C_{k-1}^{\prime}=$ $\left(E+C_{k-1} P_{k-1 \mid k-1} C_{k-1}^{\prime}\right)^{-1}$ Using this and definitions of $P_{k}, P_{k \mid k}$, we get $P_{k}^{-1}=P_{k \mid k}$.

$P_{0}^{-1} r_{0}=\hat{x}_{0 \mid 0}$ due to corollary assumption. Suppose that $P_{k-1}^{-1} r_{k-1}=\hat{x}_{k-1 \mid k-1}$. The induction hypothesis, equality $P_{k}^{-1}=P_{k \mid k}$ and (19) imply $(E+$ $\left.C_{k-1} P_{k-1 \mid k-1} C_{k-1}^{\prime}\right)^{-1} C_{k-1} \hat{x}_{k-1 \mid k-1}=C_{k-1}\left(C_{k-1}^{\prime} C_{k-1}+\right.$ $\left.P_{k-1}\right)_{k-1}^{-1} r_{k-1}$. Combining this with definitions of $\hat{x}_{k \mid k}$, $r_{k}$ we obtain $\hat{x}_{k \mid k}=P_{k}^{-1} r_{k}$. This concludes the proof.

\section{Conclusion}

We describe a set-membership state estimation approach for a linear operator equation with uncertain disturbance restricted to belong to a convex bounded closed subset of abstract Hilbert space. It is based on the notion of an a posteriori set [16] $\mathscr{G}(y)$, informational set [5] and the notion of the minimax observable subspace for the pair $(L, H)$. The latter is new for the set-membership state estimation framework. It leads to nontrivial new results in set-membership state estimation for linear non-causal DAEs: we present new equations describing the dynamics of the minimax recursive estimator for discrete-time non-causal DAEs. We prove that these equations are consistent with the main results already established for regular DAEs. We illustrate benefits of considering non-causality in the state equation, applying our approach to a linear filtration problem with unbounded noise.

\section{Acknowledgements}

It is a pleasure to thank Prof. A.Nakonechny and Dr. V.Pichkur for insightful discussions. I also thank to Drs. V.Mallet, J.A. Hosking and my anonymous referees for their help with improving the presentation of this paper.

\section{References}

[1] A. Albert. Regression and the Moor-Penrose pseudoinverse. Acad. press, N.Y., 1972.

[2] E. Angel and R. Bellman. Dynamic programming and partial differential equations. Acad. press N.Y., 1972.

[3] T. Başar and P. Bernhard. $H_{\infty}$-optimal Control and Related Minimax Design Problems. Springer, 1995.

[4] A. Balakrishnan. Applied functional analysis. Springer, 1976.

[5] J. S. Baras and A.B. Kurzhanski. Nonlinear filtering: The set-membership and the $H_{\infty}$ techniques. In Proc. 3rd IFAC Symp.Nonlinear Control Sys.Design. Pergamon, 1995.

[6] D. Bender and A. Laub. The linear-quadratic optimal regulator for descriptor system: discrete-time case. Automatica, 23:71-85, 1987.

[7] D.P. Bertsekas and I. B. Rhodes. Recursive state estimation with a set-membership description of the uncertainty. IEEE Trans. Automat. Contr., AC-16:117-128, 1971.

[8] Z. Deng and Liu Y. Descriptor Kalman estimators. Int. J. of System Science, 30:1205-1212, 1999.

[9] I. Ekeland and R. Temam. Convex analysis and variational problems. North-Holland, Amsterdam, 1976.

[10] J.Y. Ishihara, M.H. Terra, and J.C.T. Campos. Optimal recursive estimation for discrete-time descriptor systems. Int. J. of System Science, 36(10):1-22, 2005.

[11] T. Kato. Perturbation theory for linear operators. SpringerVerlag, 1966.

[12] A. Kurzhanski and I. Valyi. Ellipsoidal Calculus for Estimation and Control. Birkhäuser, Boston, 1997.

[13] F. Lewis. A survey of linear singular systems. Circuits Systems Signal Process, 5(1), 1986.

[14] M. Milanese and R. Tempo. Optimal algorithms theory for robust estimation and prediction. IEEE Trans. Autom. Contr., 30(8):730-738, Aug 1985.

[15] M. Milanese and A. Vicino. Optimal estimation theory for dynamic systems with set membership uncertainty: An overview. Automatica, 27:997-1009, 1991.

[16] A. Nakonechny. Minimax estimation of functionals defined on solution sets of operator equations. Arch.Math. 1, Scripta Fac. Sci. Nat. Ujer Brunensis, 14:55-60, 1978.

[17] R. Nikoukhah, S.L. Campbell, and F. Delebecque. Kalman filtering for general discrete-time linear systems. IEEE Transactions on Automatic Control, 44:1829-1839, 1999.

[18] R. Rockafellar. Convex analysis. Princeton, 1970.

[19] A. Sayed. A framework for state-space estimation with uncertain models. IEEE Trans. Autom. Contr., 46:998-1013, 2001.

[20] J.S. Shamma and Tu Kuang-Yang. Set-valued observers and optimal disturbance rejection. IEEE Trans. Autom. Contr., 44(2):253-264, Feb 1999.

[21] R. Tempo. Robust estimation and filtering in the presence of bounded noise. IEEE Trans. Autom. Contr., 33(9):864-867, 1988. 
[22] S. Xu and J. Lam. Reduced-order $H_{\infty}$ filtering for singular systems. System \& Control Letters, 56(1):48-57, 2007.

[23] H. Zhang, L. Xie, and Y. Soh. Risk-sensitive filtering, prediction and smoothing for discrete-time singular systems. Automatica, 39:57-66, 2003.

[24] S. Zhuk. Closedness and normal solvability of an operator generated by a degenerate linear differential equation with variable coefficients. Nonlin. Oscillations, 10:1-18, 2007.

[25] S. Zhuk. State estimation for a dynamical system described by a linear equation with unknown parameters. Ukr. Math. J., 61(2):178-194, 2009. 